\title{
Identificación morfofisiológica de hongos en genotipos de maíz
}

\author{
(Identification of fungi in corn genotypes)
}

\author{
José Luis Arispe V. ${ }^{1}$, Abiel Sánchez A. ${ }^{1 *}$, Ma. Elizabeth Galindo C. ${ }^{1}$, \\ Mario Ernesto Vázquez B. ${ }^{1}$, Arnoldo Oyervides G. ${ }^{1}$, Raúl Rodríguez G. ${ }^{2}$ \\ ${ }^{1}$ Universidad Autónoma Agraria Antonio Narro, Buenavista, Saltillo, Coahuila. \\ ${ }^{2}$ INIFAP General Terán. Campo Experimental General Terán, Nuevo León. \\ *Autor para correspondencia: abielsanchez@hotmail.com
}

RECIBIDO: 19 de Julio de 2019

APROBADO: 24 de Septiembre de 2019

DOI: 10.22370/bolmicol.2019.34.2.1801

LOS AUTORES DECLARAN NO TENER CONFLICTO DE INTERESES

Palabras claves: Semilla; Incidencia; Enfermedad; Cultivo.

Key words: Seed; Incidence; Disease; Crop.

\section{RESUMEN}

El cultivo de maíz es la base de la alimentación para México, el objetivo de este trabajo fue identificar la micobiota en cuatro genotipos de maíz de Saltillo, Coahuila y cuatro de Tepalcingo, Morelos. Se realizó de acuerdo a la prueba papel secante y congelamiento, se tomaron 1000 semillas de maíz por cada genotipo, las cuales se desinfectaron con hipoclorito de sodio al $3 \%$ (2 veces) y posteriormente se enjuagaron con agua destilada por 1 min. ( 2 veces). La siembra fue realizada en charolas de plástico 18.5 x $25 \mathrm{~cm}$, sobre papel secante estéril previamente humedecido, las charolas se mantuvieron a temperatura ambiente de 26 ${ }^{\circ} \mathrm{C} \pm 2{ }^{\circ} \mathrm{C}$ durante 11 días en la cámara bioclimática del Laboratorio de Fitopatología de la Universidad Autónoma Agraria Antonio Narro. Terminado el periodo de la incubación, se procedió a contar y aislar el número de las colonias de hongos por su color por repetición, para su posterior purifica- ción e identificación, así como las semillas sanas, es decir, aquellas que no presentaron crecimiento de micelio y la incidencia reportándose como porcentaje de semilla colonizada, analizando los datos en el programa de la Universidad de Nuevo León versión 2.5. Se observó diferencia estadística entre la incidencia de hongos en los genotipos de maíz P $>$ F 0.00, con un coeficiente de variación del $22.77 \%$, los genotipos de Tepalcingo con una media del 77.925\% y Saltillo $87.725 \%$, reportando por primera vez a Acremonium sp. en Saltillo, Coahuila y Tepalcingo, Morelos, México.

\section{ABSTRACT}

Corn crop is the basis of the diet for Mexico; the objective of this work was to identify the mycobiota in four corn genotypes from Saltillo, Coahuila and four from Tepalcingo, Morelos. It was carried out according to the secant and freezing test, 1000 corn seeds were taken of each 
genotype, which were disinfected with $3 \%$ sodium hypochlorite (2 times) and then rinsed with distilled water for $1 \mathrm{~min}$. (2 times). Sowing was carried out in 18.5 x $25 \mathrm{~cm}$ plastic trays, on sterile blotting paper previously moistened, the trays were kept at room temperature $26^{\circ} \mathrm{C} \pm 2{ }^{\circ} \mathrm{C}$ during 11 days in the bioclimatic chamber of the Phytopathology Laboratory of the Autonomous University Agraria Antonio Narro, after the incubation period, we proceeded to count and isolate the number of fungal colonies by their color by repetition, for further purification and identification, as well as healthy seeds, that is, those that did not present mycelial growth and incidence was reported as percent of colonized seed, data were analyzed in the program of the University of Nuevo Leon version 2.5. Statistical difference was observed between the incidence of fungi in corn genotypes $\mathrm{P}>\mathrm{F} 0.00$, with a coefficient of variation of $22.77 \%$. Tepalcingo genotypes with an average of $77.925 \%$ and Salti1lo $87.725 \%$. It is reported for the first time Acremonium sp. in Saltillo, Coahuila and Tepalcingo, Morelos, Mexico.

\section{INTRODUCCION}

En el maíz la pudrición de tallo y mazorca está asociado con Fusarium verticillioides y Fusarium graminearum ${ }^{1}$.

En México los principales géneros de hongos que intervienen en la pudrición de la mazorca son; Diplodia, Giberella, Fusarium, Aspergillus ${ }^{2}$ y Penicillium ${ }^{3}$, siendo las principales especies $F$. verticillioides, $F$. graminearum, $F$. proliferatum y F. subglutinams ${ }^{4}$. Sin embargo, $F$. verticillioides la especies más importante con amplia distribución en todas las áreas tropicales y subtropicales donde se cultiva maíz, por ocasionar la pudrición del tallo y de la mazorca 5 .

Las principales enfermedades que atacan al maíz en México son de origen fúngico, están dis- tribuidas en todo el país y su aparición está sujeta a las condiciones ambientales que favorecen la infección y multiplicación del patógeno, así como a la fuente de inóculo y la susceptibilidad de los genotipos ${ }^{6}$.

Las enfermedades fúngicas provocan pérdidas anualmente en las zonas maiceras, se reportan aproximadamente 125 enfermedades causantes de éstas, para su reconocimiento y manejo se clasifican de acuerdo a la parte de la planta que infectan, como: follaje, espiga, tallo y mazorca ${ }^{7}$.

\section{MATERIALES Y MÉTODOS}

\section{Ubicación del Experimento}

El presente trabajo se realizó en el laboratorio de Fitopatología del Departamento de Parasitología, de la Universidad Autónoma Agraria Antonio Narro.

\section{Material Genético}

Se utilizaron los genotipos VS-221, Criollo, Cafime y Jaguan en Saltillo, y H-515, Zapata 7, H-507 y un Criollo en Tepalcingo, los cuales tenían 2 meses en almacenamiento.

\section{Prueba papel secante y congelamiento}

Se realizó de acuerdo a la prueba del manual de laboratorio para la semilla de maíz y trigo ${ }^{8}$, modificada, en este estudio se tomaron 1000 semillas por genotipo. Se tomaron 50 semillas de maíz por repetición dando un total de 20 repeticiones por genotipo, las cuales se desinfectaron con hipoclorito de sodio al $3 \%$ (2 veces) y posteriormente se enjuagaron con agua destilada por $1 \mathrm{~min}$. (2 veces).

La siembra fue realizada en charolas de plástico 18.5 x $25 \mathrm{~cm}$, sobre dos capas de papel secante estéril previamente humedecido, 50 semillas fueron seleccionadas y distribuidas uniformemente en la charola, finalmente éstas fueron rotuladas 
y selladas con cinta parafilm para su fácil identificación.

Las charolas se mantuvieron a temperatura ambiente de $25^{\circ} \mathrm{C} \pm 2{ }^{\circ} \mathrm{C}$ durante 2 días en la cámara bioclimática del Laboratorio de Fitopatología, posteriormente se mantuvieron en el congelador del Laboratorio de Semillas del Departamento de Producción a $-20{ }^{\circ} \mathrm{C} \pm 2{ }^{\circ} \mathrm{C}$ durante $24 \mathrm{~h}$, finalmente se retiraron del congelador y se mantuvieron a una temperatura ambiente de $26{ }^{\circ} \mathrm{C} \pm 2$ durante 11 días, alternando $12 \mathrm{~h}$ luz blanca y $12 \mathrm{~h}$ de oscuridad.

\section{Aislamiento de los patógenos}

Una vez observado el desarrollo completo de las diferentes colonias fungicas en las charolas se procedió a aislar los patógenos, tomando una muestra de micelio con una aguja de las diferentes repeticiones y colocándolo en placas de Petri con PDA para su desarrollo, incubándose a una temperatura de $28^{\circ} \mathrm{C}$.

\section{Purificación de los patógenos mediante cultivos monoconidiales}

De las cepas de los hongos se extrajeron explantes de $5 \mathrm{~mm}$ de diámetro y se depositaron en tubos de ensayo con $9 \mathrm{~mL}$ de agua destilada estéril y con una micropipeta se extrajo $1 \mathrm{~mL}$ y se vertió en medio PDA sintético y con una varilla estéril se dispersó, pasado $24 \mathrm{~h}$ se extrajo un solo conidio germinado y se colocó en las placas de Petri con medio de cultivo PDA, las cuales se incubaron a 26 ${ }^{\circ} \mathrm{C} \pm 2{ }^{\circ} \mathrm{C}$ por 10 días.

\section{Identificación de los patógenos}

Con una aguja de disección se tomó una pequeña porción del hongo y se colocó en un portaobjetos con lactofenol y se observó en el microscopio compuesto en aumento de 10 y $40 \mathrm{X}$ y para la identificación morfológica se utilizaron los manuales de Barnett and Hunter ${ }^{9}$ y Warham ${ }^{8}$.

\section{Evaluación final}

La incidencia se reportó como porcentaje de semilla colonizada por los patógenos en cada repetición por genotipo.

\section{Análisis estadístico}

Los resultados obtenidos de la incidencia de los hongos en la semilla de maíz, fueron sometidos al análisis de varianza y prueba de separación de medias mediante Tukey al 0.05 de significancia, para detectar diferencia entre tratamientos, en el programa estadístico de Nuevo León.

\section{RESULTADOS Y DISCUSIÓN}

Se observa diferencia estadística entre la incidencia de hongos en los genotipos de maíz $\mathrm{P}>\mathrm{F}$ 0.00 , con un coeficiente de variación del $22.77 \%$, los genotipos de Tepalcingo con una media del $77.925 \%$ y Saltillo $87.725 \%$ (Tabla 1 ).

Tabla 1. Incidencia de hongos en los genotipos de maíz. Letras iguales no son estadísticamente diferentes de acuerdo a la prueba de Tukey $(\mathrm{P}<0.05)$.

\begin{tabular}{|c|c|c|}
\hline GENOTIPO & MEDIA \% & \multicolumn{2}{|c|}{ AGRUPACION } \\
\hline VS-221 & 95.9 & A \\
\hline Jaguan & 95.8 & A \\
\hline Zapata 7 & 92.2 & \multicolumn{2}{|c|}{ AB } \\
\hline Criollo Tepalcingo & 90.8 & \multicolumn{2}{|c|}{ ABC } \\
\hline H-515 & 85.2 & \multicolumn{2}{c|}{ C } \\
\hline Criollo Saltillo & 83.1 & \\
\hline Cafime & 76.1 & \\
\hline H-507 & 43.5 & \\
\hline
\end{tabular}


En las figuras 1 y 2 se muestra los hongos identificados en las semillas de la región de Saltillo y Tepalcingo, así como la incidencia en que estuvieron presentes, mencionan a los géneros de Fusarium, Aspergillus y Penicillium como los hongos más toxicogénicos de mayor importancia con amplia distribución geográfica ${ }^{10,11,12}$ y que pueden desarrollarse sobre una amplia gama de granos almacenados y semillas de maíz, en cambio hongos de mayor importancia que causan pudrición de la mazorca reporta a Diplodia, Gibberella, Fusarium y Aspergillus $^{13}$.

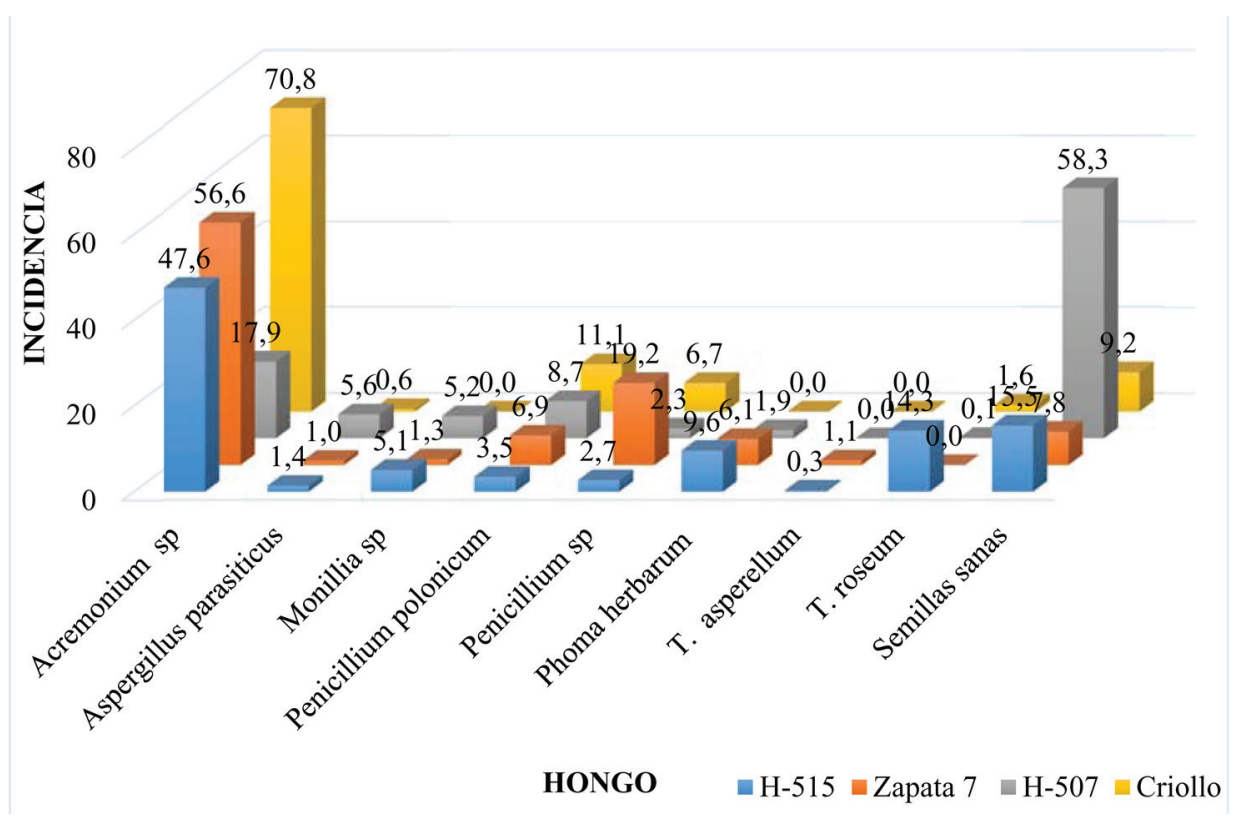

Figura 1. Incidencia de hongos en genotipos de Tepalcingo, Morelos.

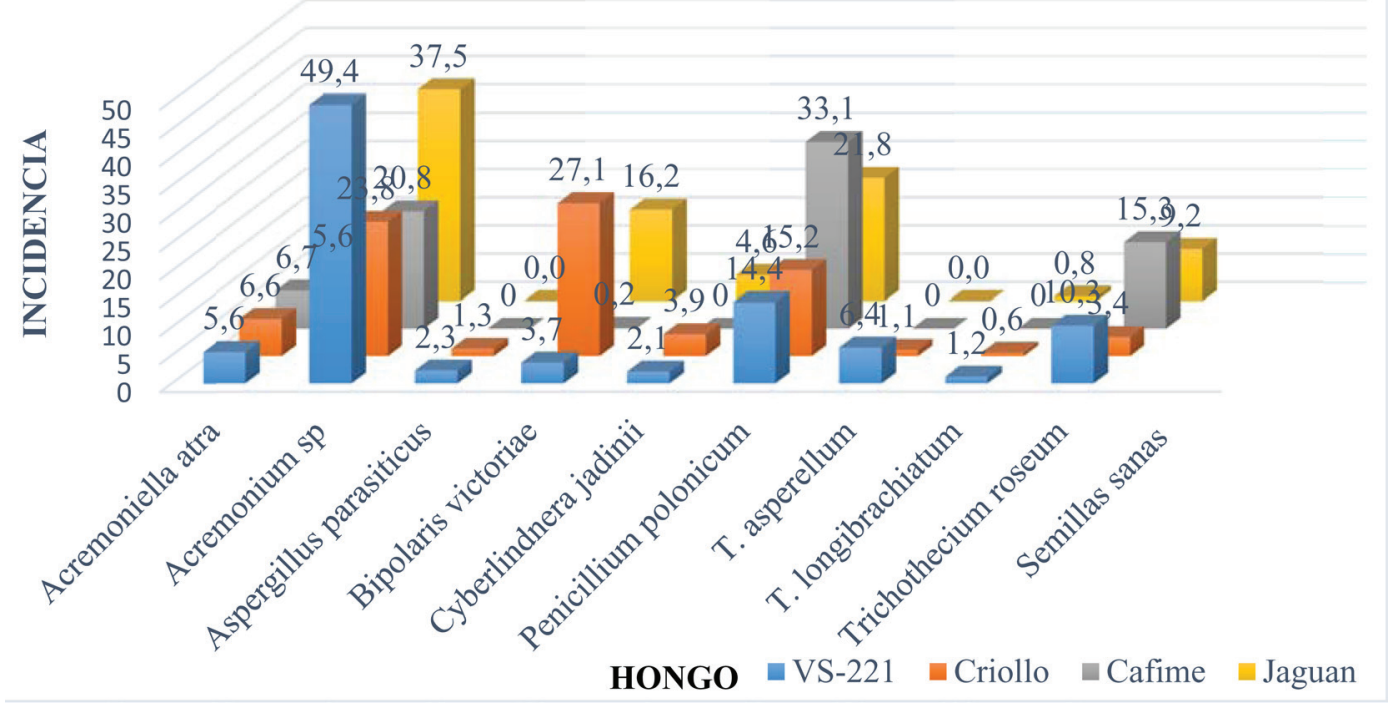

Figura 2. Incidencia de hongos en genotipos de Saltillo, Coahuila. 
El principal agente patógeno causante de la podredumbre de la mazorca de maíz es $F$. verticillioides ${ }^{14}$, sin embargo, en estos ocho genotipos no estuvo presente, pero se reporta por primera vez a Acremonium sp. (Figuras 3 y 4) con una incidencia del 20.8 al $49.4 \%$ en Saltillo y 17.9 al $70.8 \%$ en Tepalcingo, se reportó a Acremonium zeae como un endófito protector del maíz, capaz de inhibir a Aspergillus flavus y a F. verticillioi$d e s^{15}$, sin embargo, es considerado un patógeno en el cultivo de maíz. Se reportó la necrosis vascular causada por Cephalosporium acremonium y marchitez tardía, causada por Cephalosporium maydis, con síntomas parecidos a los de F. verticillioides ${ }^{16}$, además en esta investigación estuvieron presentes los géneros Penicillium y Aspergillus en un promedio por los 4 genotipos en Saltillo de $21.13 \%$ y 0.9 $\%$ y en Tepalcingo con $15.27 \%$ y $2.2 \%$, siendo el genotipo Jaguan con la mayor incidencia en Salti1lo $95.77 \%$ y en Tepalcingo el Criollo con $90.8 \%$.
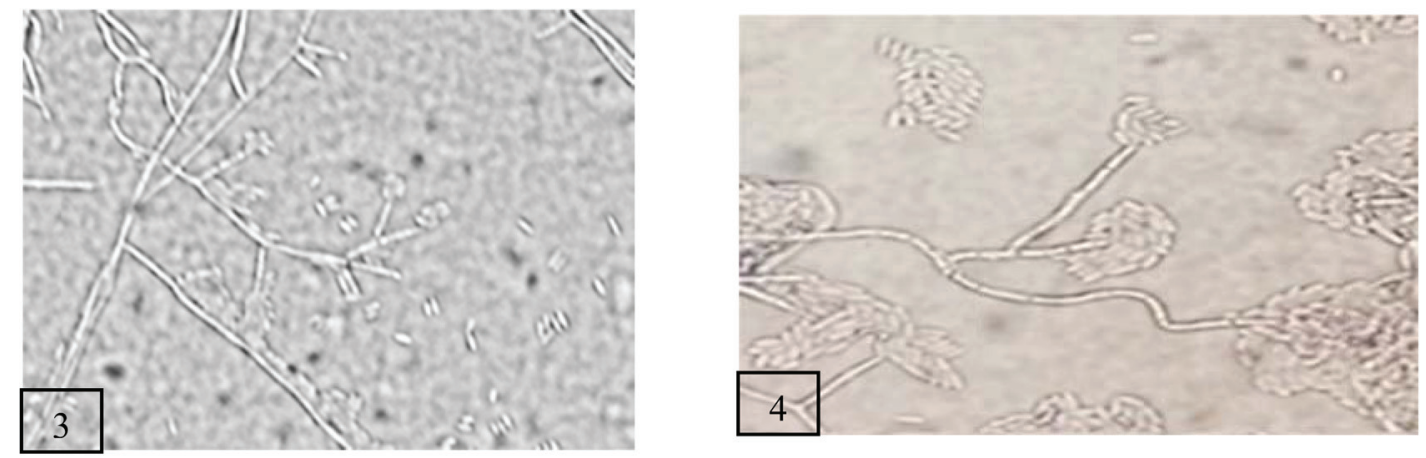

Figuras 3 y 4. Micelio hialino y septado con conidóforos largos, los cuales terminan en conidios agrupados simulando una cabeza.

\section{AGRADECIMIENTOS}

A la empresa Agrícola el Caudillo por proveer los genotipos de Tepalcingo, Morelos y a la Universidad Autónoma Agraria Antonio Narro por los de Saltillo, Coahuila.

\section{REFERENCIAS}

1. González, H. A., Vázquez, G. L.M., Sahagún, C. J., Rodríguez, P. J. E. y Pérez, L. D. J. Rendimiento del maíz de temporal y su relación con la pudrición de mazorca. Agric. Téc. Méx. 2007; 33 (1 y 2): 33-42.

2. DuPont Pioneer [Internet]. México: DuPont Pioneer; 2014 [citado 11 ene 2019]. Pudrición de mazorca; p: 1,2. Disponible en: https://www.pioneer.com/web/site/mexico/articulos/centronorte/

3. Agrosíntesis [Internet]. México: Editorial Agro Cultivos; 2010 [citado 7 mar 2019]. Pudrición de la mazorca [Aprox. 1 pantalla]. Disponible en: https:// www.agrosintesis.com/pudricion-de-la-mazorca-2/

4. PDL, 2018. Plant Disease Library. Pudrición de la mazorca por Penicillium y Fusarium. Disponible en: https://plantix.net/plant-disease/en. [Consultado el 24 de marzo de 2019].

5. McGee, D. C. Maize Disease: a reference source for seed technologists. 2 edition. Minnesota: American Phytopathological Society; 1988.

6. Varón de Agudelo, F y Sarria-Villa, C. A. Enfermedades del maíz y su manejo: compendio ilustrado. 1 edición. Palmira, Colombia: editor ICA; 2007. 
7. Rodríguez, M. R. y De León, C. El cultivo del maíz: temas selectos. México: D.F. COLPOS: Mundi-Prensa México; 2008.

8. Warham, EJL, Bulter D. y Sulton, B. C. Ensayos para la semilla de maíz y trigo: manual de laboratorio. México: Centro Internacional de Mejoramiento de Maíz y Trigo CIMMYT; 2003.

9. Barnett, L. H. and Hunter, B. B. Illustrate genera of imperfect fungi. Minnesota: The American Phytopathology Society Press; 2006.

10. Richardson, M. An annotated list of seed-borne diseases. England: CAB; 1979.

11. Christensen, C. y Kaufmann, H. Contaminación por hongos en granos almacenados. México: Editorial Pax; 1976.

12. Sauer, O. Identification of fungi in grains and feeds. U.S.A: Department of Agriculture; 1984.

13. Peiretti, U. D. A., Nazar, L. M. C., Biasutti, V. C. A., Giorda, L. L. M. Susceptibilidad a Fusa- rium verticillioides (Sacc.) Nirenberg en la población de maíz MPB-FCA 8561. Agronomía Mesoamericana [Internet]. 2007;18(2):171-176.

14. García, D. A. Centro Internacional de Mejoramiento de Maíz y Trigo (CIMMYT). México, 2016. Disponible en: http://conservacion.cimmyt. org/es/component/content/article/88-boletin-enlace-/2069-productores-identifican-hongos-que-causan-pudricion-de-mazorca. [Citado 21 ene 2019].

15. Wicklow, D. T., Roth S., Deyrup, S. T., Gloer, J. B. A protective endophyte of maize: Acremonium zeae antibiotics inhibitory to Aspergillus flavus and Fusarium verticillioides. Mycol. Res. [Internet]. 2005[Consultado el 26 de marzo de 2019]; 109(5): 610-618. Disponible en: https:// doi.org/10.1017/S0953756205002820.

16. Programa de Maíz del CIMYYT. Enfermedades del maíz: una guía para su identificación en el campo [Internet]. Cuarta edición. México, D.F.: Centro Internacional de Mejoramiento de Maíz y Trigo CIMYYT; 2004 [Citado 12 mar 2019]. Disponible en: https://repository.cimmyt.org/xmlui/ bitstream/handle/10883/812/94349.pdf. 